\title{
Sampling-based Multi-robot Motion Planning
}

\author{
Zhi Yan, Nicolas Jouandeau and Arab Ali Cherif \\ Advanced Computing Laboratory of Saint-Denis (LIASD) \\ Paris 8 University \\ 93526 Saint-Denis, France \\ $\{y z, n, a a\} @$ ai.univ-paris8.fr
}

Keywords: Multi-robot systems, Coordination, Motion planning, Sampling-based method.

\begin{abstract}
This paper describes a sampling-based approach to multi-robot motion planning. The proposed approach is centralized, which aims to reduce interference between mobile robots such as collision, congestion and deadlock, by increasing the number of waypoints. The implementation based on occupancy grid map is decomposed into three steps: the first step is to identify primary waypoints by using the Voronoi diagram, the second step is to generate additional waypoints by sampling the Voronoi diagram, and the last step is to assign the waypoints to robots by using the Hungarian method. The approach has been implemented and tested in simulation and the experimental results show a good system performance for multi-robot motion planning.
\end{abstract}

\section{INTRODUCTION}

In robotics, the motion planning problem is to produce a continuous motion from one configuration to another in configuration space, while avoiding collision with obstacles. The motion is represented as a path in configuration space. Motion planning is eminently necessary for mobile robot since, by definition, a robot accomplishes tasks by moving in the real world (Latombe, 1991). Environments for autonomous mobile robot are usually represented by an occupancy grid map. For most of motion planning algorithms, the robot is reduced to a point in a twodimensional plane.

Multi-robot motion planning is one of the most challenging tasks in multi-robot systems. It should consider not only the obstacles (whether static or dynamic) in the environment, but also the possible interference between robots. When robots in a team are used to perform independent tasks in a shared workspace, each one will become a mobile obstacle for the other. Thus each robot should take into account the movement of other robots. One well-studied example is the multi-robot space sharing problem (Jäger and Nebel, 2001) (Luo et al., 2003) (Marcolino and Chaimowicz, 2009a) (Marcolino and Chaimowicz, 2009b) (Luna and Bekris, 2011).

Multi-robot environment must definitely be dynamic, in which motion planning is inherently difficult. Even for a simple case in two dimensions, the planning is NP-hard and is not solvable in polynomial time (Guo and Parker, 2002). A very common problem in the motion planning for a group of mobile robots is when multiple robots move to the same waypoint, causing collision, congestion and deadlock, and thus affects system performance. We define this kind of dynamic standstill of system caused by waypoint conflict as the multi-robot waiting situation problem. Because, for better or worse, a robot should wait until the other pass first, or all robots should wait for planner to replan their local trajectories. Therefore, some kind of mechanism is needed to coordinate the motion of robots. In this paper, we present a sampling-based approach based on centralized decision making mechanism to multi-robot motion planning. The basic idea of the proposed approach is to reduce the problem of waiting situation between robots by increasing the number of waypoints. The implementation includes three critical steps:

- The first step is toward identifying primary waypoints on top of an occupancy grid map by using the Voronoi diagram.

- The second step is generating additional waypoints on top of the Voronoi diagram by using a sampling-based method.

- The third step is toward assigning the waypoints to robots by using the Hungarian method.

The remainder of the paper is organized as follows: Section 2 describes an overview of some related 
works; Section 3 describes our sampling-based approach; Section 4 describes the experimental results obtained with our approach; and the paper is concluded in Section 5 at last.

\section{RELATED WORK}

The major approaches to multi-robot motion planning are expanded from the results of single-robot system. (Bennewitz et al., 2001) presented an approach based on $\mathrm{A}^{*}$ path planning algorithm to optimize the priorities for decoupled and prioritized path planning methods for groups of mobile robots. Their approach is a randomized method which repeatedly reorders the robots to find a sequence for which a plan can be computed and to minimize the overall path lengths. (Guo and Parker, 2002) proposed an approach based on D* path planning algorithm which contain two modules: path planning and velocity planning. The detailed process can be described as follows: first, each robot plans its own path independently, then a coordination diagram is constructed based on collision checks among all robot paths. One approach named artificial potential field (APF) is used extensively for multirobot formation control which is usually linked with multi-robot motion planning problem. (Tanner and Kumar, 2005) presented a strategy which can ensure almost global asymptotic convergence of the robots to a particular oriented formation shape, while guaranteeing collision avoidance in the process.

The approaches often used for high-dimensional motion planning problems can also be efficiently applied to deal with low-dimensional problems. There are two major families of approaches known as cell decomposition and skeletonization. The cell decomposition method often applied for multi-robot area coverage problem. (Hazon et al., 2006) presented an online robust multi-robot spanning tree coverage (ORMSTC) algorithm based on an approximate cellular decomposition for covering an unknown environment. The algorithm is called online because in which the robots do not have apriori knowledge of the work area, and it is robust and complete because in which as long as a single robot is able to move, the coverage will be completed. A typical skeletonization method is Voronoi diagram which is often applied to the problem of robotic exploration. (Wurm et al., 2008) presented a strategy for coordinating a team of exploring robots by using Voronoi diagram for the segmentation of the environment map. The strategy extracts the critical points (Thrun, 1998) as the exploration targets to assign to robots. An alternative to the Voronoi diagrams is the probabilis- tic roadmap (PRM) (Kavraki et al., 1996) which is a sampling-based method. (Švestka and Overmars, 1995) presented a PRM-based approach for multiple nonholonomic car-like robots motion planning in the same static workspace, in which the roadmaps for the composite robot are derived from roadmaps for the underlying simple robots, and the latter is computed by a probabilistic single-robot learning method.

The sampling-based methods are currently considered state-of-the-art for robot motion planning in complex environments. Because unlike the incremental heuristic search (such as $\mathrm{A}^{*}$ and $\mathrm{D}^{*}$ ) and the topological map (such as Voronoi diagram) methods, their running time does not grow exponentially with the dimension of the configuration space, but also easier to implement. Yet, the sampling-based methods are probabilistically complete, and can not determine if no solution exists.

\section{OUR APPROACH}

\subsection{Voronoi Diagram}

The first step of our approach is to identify primary waypoints on top of the occupancy grid map by applying the Voronoi diagram. The Voronoi diagram is a very popular graph partitioning method for the problem of map segmentation (Thrun, 1998) (Zivkovic et al., 2006) (Wurm et al., 2008). For a given map $m$, we define for each point $p$ in the free space $S$ of $m$, a set $O_{p}(m)$ which is made up of closest obstacle points to $p$. While the Voronoi diagram can be represented by a graph $G(m)=(V, E)$, where $V$ is the set of points in $O_{p}(m)$ that are equidistant to at least two nearest obstacles, and $E$ is the set of edges connecting two adjacent points in $V$ of $m$ :

$$
\begin{gathered}
V=p \in S|| O_{p}(m) \mid \geq 2 \\
E=(p, q) \mid p, q \in V, p \text { adjacent to } q
\end{gathered}
$$

Figure 1 shows the Voronoi diagrams of two experimental maps.

Then the topological map is built based on the Voronoi diagram. The grid map is decomposed into a small set of regions separated by narrow passages. These narrow passages, which are called critical lines, are found by analyzing a skeleton of the environment. The partitioned map is mapped into an isomorphic graph, where nodes correspond to regions and arcs connect neighboring regions. This graph is the topological map (Thrun, 1998).

On the basis of the topological map, a series of points called critical points is generated, at which the 


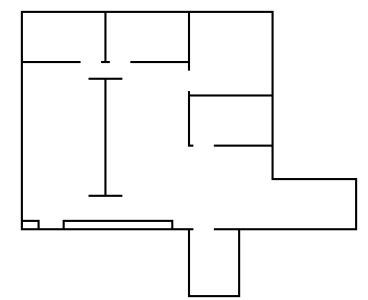

(a) map $\mathrm{A}$

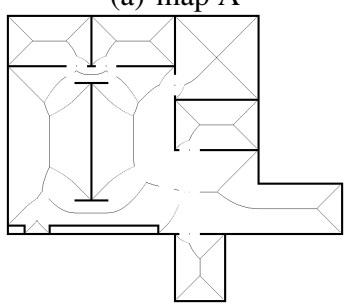

(c) map A

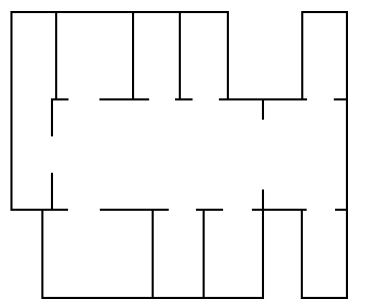

(b) map B

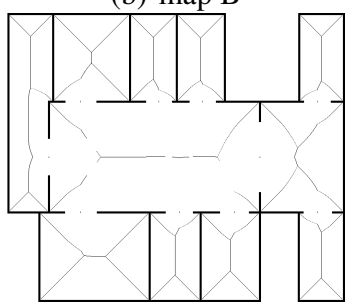

(d) map B
Figure 1: Voronoi diagrams of two experimental maps. (a) and (b) depict the original maps. (c) and (d) depict the generated Voronoi diagrams.

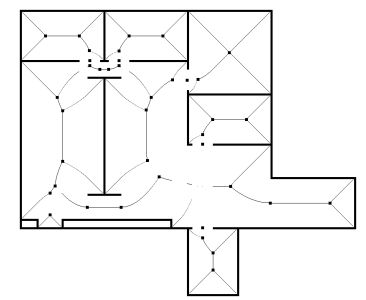

(a) map $\mathrm{A}$

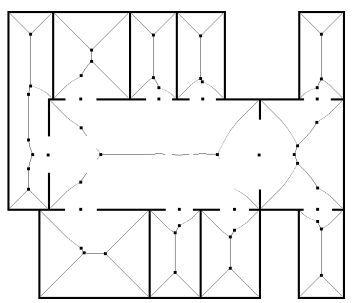

(b) map B
Figure 2: The primary waypoints extracted by using the Voronoi diagram.

distance to the nearest obstacle in the map is a local minimum. In other words, the critical points are the midpoints of the critical lines. These points are considered as the primary waypoints for mobile robot motion planning. Figure 2 illustrates the results of the first step.

\subsection{Sampling-based Method}

The second step of the approach is to generate additional waypoints on top of the Voronoi diagram by applying a PRM-like method. The original PRM is presented by (Kavraki et al., 1996), of which the basic algorithmic framework can be summarized as follows:

- Firstly, randomly sample a sufficient number of points in the configuration space (denoted by $C$ ) of the robot, keeping any that are not in collision with obstacles (denoted by $C_{o b s}$ ). This creates a point set in the the free configuration space (denoted by $C_{\text {free }}$ ).

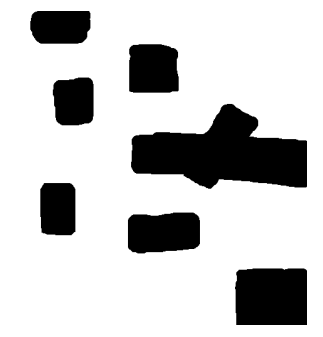

(a)

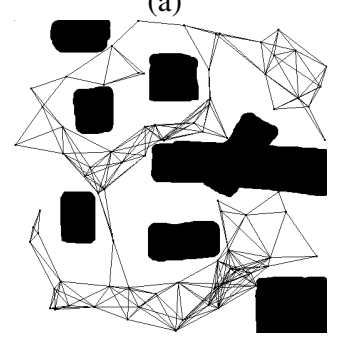

(c)

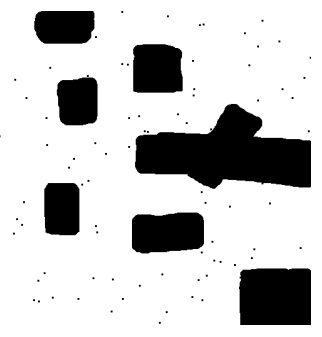

(b)

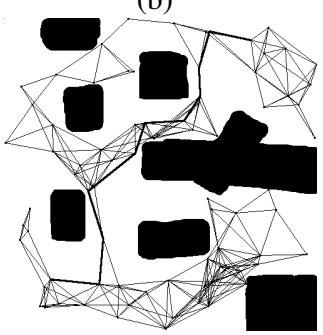

(d)
Figure 3: Generation of the probabilistic roadmap. (a) shows a configuration space in which we will build a probabilistic roadmap. (b) shows the random points generated in $C_{\text {free }}$. (c) shows the roadmap constructed by connecting the samples relatively close using the straight line planner. (d) shows a path found by querying the constructed roadmap using the $\mathrm{A}^{*}$ algorithm.

- Secondly, using a local planner (such as straight line planner), attempt to connect pairs of samples that are relatively close to each other by thoroughly sampling and collision checking configurations between them. This creates a graph data structure called a roadmap.

- Finally, to query the roadmap, first attempt to connect the start and goal configurations to the existing graph. If that is successful, search the graph for a path from start to goal using any standard graph search method (such as A* algorithm).

Like most of motion planning algorithms, the PRM-like method often computes a collision free roadmap in a configuration space where the object is reduced to a point. This point represents the robot's model in the environment and the dimension of configuration space is equal to the number of degrees of freedom of the mechanical system. Then the algorithm searches a free path for a point in the roadmap. Figure 3 illustrates the process of generating a probabilistic roadmap for an example occupancy grid map with 80 random samples.

An extension of the original PRM, which is called medial axis based PRM (i.e. MAPRM) (Wilmarth et al., 1999), aims to efficiently retract any sampled configuration, free or not, onto the medial axis of the free space without having to compute the medial axis explicitly. This strategy is applied to our approach 


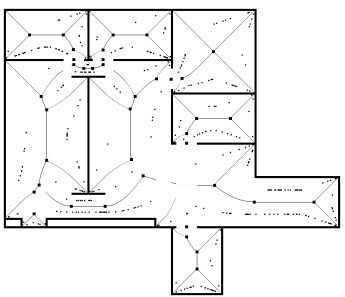

(a) $\operatorname{map} \mathrm{A}$

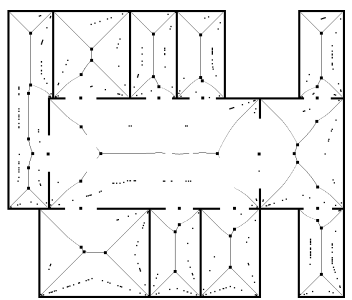

(b) map B
Figure 4: The additional waypoints extracted by using the sampling-based method with 400 random samples.

with some small modifications. The implementation details can be seen in Algorithm 1. When the random configuration $p$ is sampled, the nearest obstacle configuration $q$ to $p$ should be found, and another configuration $r$ is set to fix $p$ at the center of $q$ and $r$. If $r$ is a free configuration, then $p$ will be moved to their center and $r$ will be pushed to a farther location. This step is repeated until $r$ becomes an obstacle configuration. The algorithm produces a self adaptive medial axis synthesis, which is fitted to the clearance of each local free space. The results of applying this algorithm can be seen in Figure 4.

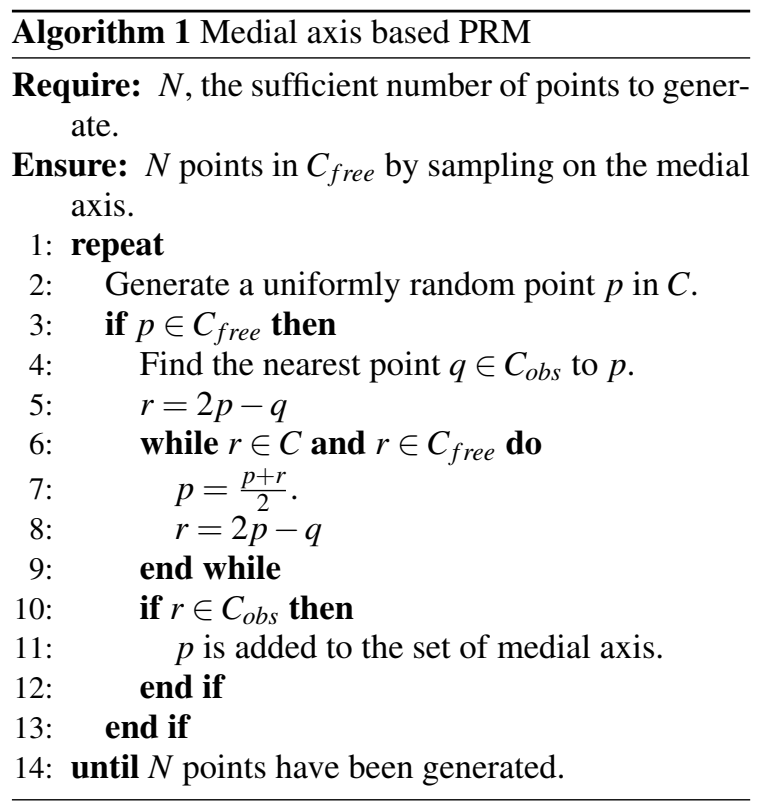

\subsection{Hungarian Method}

The last step is to assign the waypoints to robots by using the Hungarian method. The Hungarian method is a combinatorial optimization algorithm which can solve the assignment problem in polynomial time $\left(O\left(n^{3}\right)\right)$. It was developed and published by (Kuhn, 1955). In the context of our research, given $n$ way- points and $n$ robots, we would like to find the best way (cost minimizing assignment) to assign the waypoints to the robots. The implementation details on this issue by using the Hungarian method can be summarized in the following four steps:

1. Construct a $n \times n$ matrix containing the cost of assigning each waypoint to a robot. The cost could be the distance or energy required for the robot to reach the waypoint.

2. The minimum element in each row is subtracted from all the elements in that row, and the minimum element in each column is subtracted from all the elements in that column.

3. Find the minimum number of lines required to cover all the zeros across rows and columns. If the number of the lines is $n$, then the optimal waypoint allocation plan can be given by selecting a combination from the modified cost matrix in such a way that the sum is zero. Otherwise, continue with Step 4.

4. Find the smallest element which is not covered by any of the lines. Then subtract it from each element which is not covered by the lines and add it to each element which is covered by a vertical and a horizontal line. Continue with Step 3.

The implementation of the Hungarian method described above requires a square cost matrix, which means the number of waypoints and the number of robots should be equal, thus each robot can only move to a waypoint. On the contrary, if the cost matrix is not square, then we have to augment it into a square matrix by adding zero rows or columns. Suppose there are three robots, $r_{0}, r_{1}$ and $r_{2}$, and only two waypoints, $p_{0}$ and $p_{1}$, then the $3 \times 3$ cost matrix can be represented as follows:

$$
C=\left(\begin{array}{lll}
\operatorname{cost}\left(r_{0}, p_{1}\right) & \operatorname{cost}\left(r_{0}, p_{2}\right) & 0 \\
\operatorname{cost}\left(r_{1}, p_{1}\right) & \operatorname{cost}\left(r_{1}, p_{2}\right) & 0 \\
\operatorname{cost}\left(r_{2}, p_{1}\right) & \operatorname{cost}\left(r_{2}, p_{2}\right) & 0
\end{array}\right)
$$

Finally we combine the three methods described above (i.e. the Voronoi diagram, the probabilistic roadmap and the Hungarian method), and take into account both the primary waypoints and the additional waypoints to coordinate multi-robot motion planning. 


\section{EXPERIMENTS}

\subsection{Experimental Setup}

Our approach has been implemented and tested in simulation with the 2D multiple robots simulator Player/Stage (Gerkey et al., 2003). The experiment is to explore an unknown indoor environment by using a group of virtual Pioneer 2-DX robot equipped with a laser range finder which can provide 361 samples with 180 degrees field of view and a maximum range of 8 meters. Every robot is equipped with an abstract localization device which models the implementation of SLAM (Simultaneous Localization And Mapping), and a navigation device which performs the wavefront propagation algorithm (Latombe, 1991) for global path planning and the vector field histogram algorithm (Ulrich and Borenstein, 1998) for goal seeking and local obstacle avoidance. A central planning server is assumed, which is able to communicate with all robots and assign the exploration tasks to each one. In addition, all the robots share an occupancy grid map with respect to the structure of the environment during their exploration mission.

A different number of robots (from two to six) combining with two environments is used to evaluate our approach. In addition, a comparison with experimental results of our approach and a Voronoi-based approach is given. Figure 1 ((a) and (b)) depicts two experimental maps used in our simulation which are enclosed spaces with 14 meters long and 16 meters wide. The ratio between real-world time and simulation time is about 1:3. All experiments reported in this paper were carried out on a system with an Intel Core 2 Duo E8400 3.00GHz processor, an Intel Q43 Express chipset and two DDR2 $800 \mathrm{MHz} 1024 \mathrm{MB}$ dual channel memory. A screenshot of our implementation can be seen in Figure 5.

\subsection{Experimental Results}

The results of the experiments are given in Figure 6. We measured the exploration time gained by our sampling-based approach and compared to the Voronoi-based approach. In each plot, the abscissa denotes the team size of the mobile robots, the ordinate denotes the time gain in percent of the total exploration time, and the error bar indicates the confidence interval of each corresponding gain of robot team size with the 0.95 confidence level. 10 runs are performed for each team size. Figure 6 shows that an exploration time saving of $5.8 \%$ to $16.1 \%$ in map A and $6.4 \%$ to $15.3 \%$ in map $B$ is obtainable under our sampling-based approach compared to the Voronoi-

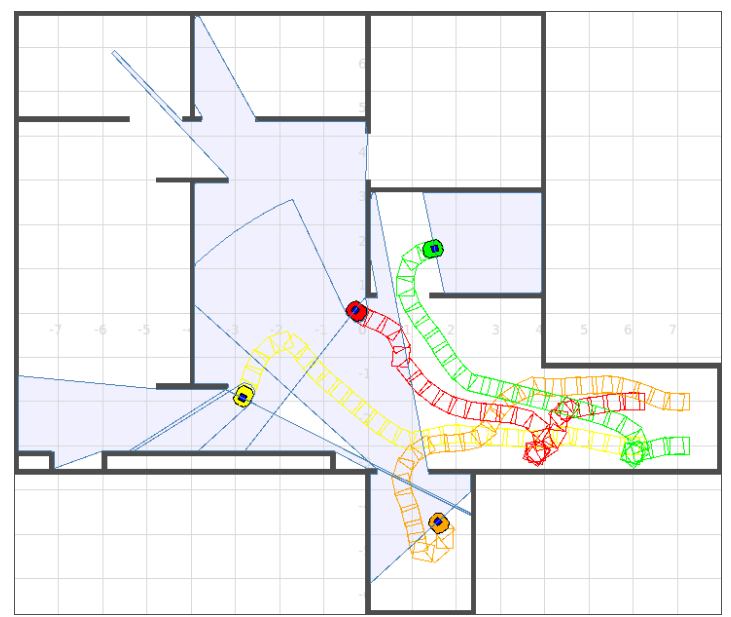

Figure 5: Four robots explore an unknown environment cooperatively. The green robot and the orange robot are exploring different rooms. The red robot and the yellow robot are exploring corridors along different paths.

Table 1: The Number of Waypoint Conflicts by Using Our Sampling-based Approach Compared with The Voronoibased Approach

(a) map A

\begin{tabular}{|l|c|c|c|c|c|}
\hline \# robots & 2 & 3 & 4 & 5 & 6 \\
\hline \hline Sampling-based & 2.5 & 2.9 & 4.3 & 3.1 & 3.4 \\
\hline Voronoi-based & 6.2 & 6.7 & 11.6 & 8.1 & 10.2 \\
\hline (b) map B \\
\begin{tabular}{|l|c|c|c|c|c|}
\hline \# robots & 2 & 3 & 4 & 5 & 6 \\
\hline \hline Sampling-based & 0.0 & 1.4 & 2.7 & 2.9 & 2.4 \\
\hline Voronoi-based & 4.1 & 6.2 & 6.4 & 7.7 & 10.7 \\
\hline
\end{tabular}
\end{tabular}

based approach. These results show that our technique could improve performance of the multi-robot motion planning.

We also counted the average number of waypoint conflicts in each experiment map, as shown in Table 1. This table shows that the waypoint conflict is obviously reduced by using our approach compared to the Voronoi-based approach. For this reason, the problem of waiting situation such as collision, congestion and deadlock has been alleviated and the system performance is improved.

\section{Conclusion}

In this paper, we presented a multi-robot motion planning approach based on sampling method. This approach is designed to relieve multi-robot waiting situation problem such as collision, congestion and deadlock, by increasing the number of waypoints for mobile robots. The proposed approach includes three 


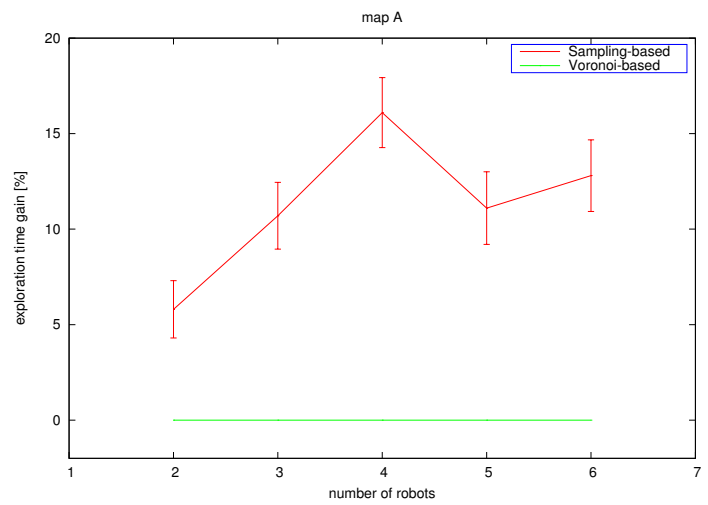

(a) map A

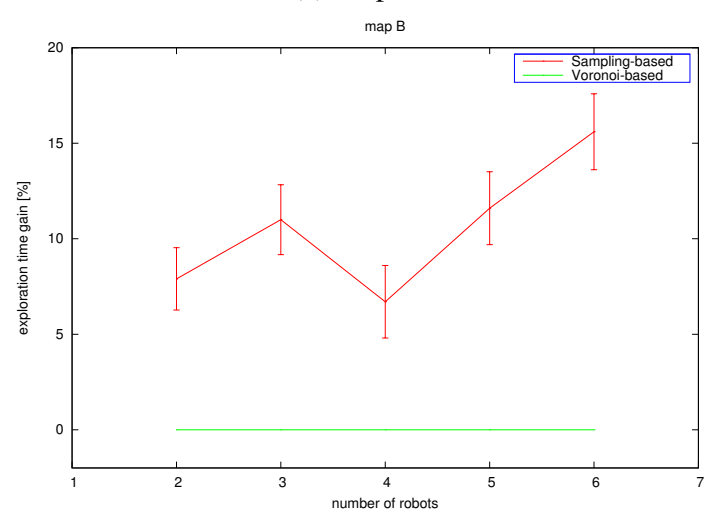

(b) map B

Figure 6: Exploration time gained by using our samplingbased approach compared with the Voronoi-based approach.

main steps: the first step is to identify primary waypoints on top of an occupancy grid map by using the Voronoi diagram, the second step is to generate additional waypoints on top of the Voronoi diagram by using the sampling-based method, and the third step is to assign the waypoints to robots by using the Hungarian method. The efficiency of our approach was verified by simulation and experimental results.

\section{REFERENCES}

Bennewitz, M., Burgard, W., and Thrun, S. (2001). Optimizing schedules for prioritized path planning of multi-robot systems. In Proceedings of ICRA'01, pages 271-276, Seoul, Korea.

Gerkey, B. P., Vaughan, R. T., and Howard, A. (2003). The Player/Stage project: Tools for multi-robot and distributed sensor systems. In Proceedings of ICAR'03, pages 317-323, Coimbra, Portugal.

Guo, Y. and Parker, L. E. (2002). A distributed and optimal motion planning approach for multiple mobile robots.
In Proceedings of ICRA'02, pages 2612-2619, Washington, DC, USA.

Hazon, N., Mieli, F., and Kaminka, G. A. (2006). Towards robust on-line multi-robot coverage. In Proceedings of ICRA'06, pages 1710-1715, Orlando, FL, USA.

Jäger, M. and Nebel, B. (2001). Decentralized collision avoidance, deadlock detection, and deadlock resolution for multiple mobile robots. In Proceedings of IROS'01, pages 1213-1219, Maui, HI, USA.

Kavraki, L. E., Kolountzakis, M. N., and Latombe, J.-C. (1996). Analysis of probabilistic roadmaps for path planning. In Proceedings of ICRA'96, pages 3020 3025, Minneapolis, MN, USA.

Kuhn, H. W. (1955). The hungarian method for the assignment problem. Naval Research Logistics Quarterly, 2(1):83-97.

Latombe, J.-C. (1991). Robot Motion Planning. Kluwer Academic Publishers.

Luna, R. and Bekris, K. E. (2011). Efficient and complete centralized multi-robot path planning. In Proceedings of IROS'11, pages 3268-3275, San Francisco, CA, USA.

Luo, C., Yang, S. X., and Stacey, D. A. (2003). Realtime path planning with deadlock avoidance of multiple cleaning robots. In Proceedings of ICRA'03, pages 4080-4085, Taipei, Taiwan.

Marcolino, L. S. and Chaimowicz, L. (2009a). Traffic control for a swarm of robots: Avoiding group conflicts. In Proceedings of IROS'09, pages 1949-1954, St. Louis, MO, USA.

Marcolino, L. S. and Chaimowicz, L. (2009b). Traffic control for a swarm of robots: Avoiding target congestion. In Proceedings of IROS'09, pages 1955-1961, St. Louis, MO, USA.

Švestka, P. and Overmars, M. H. (1995). Coordinated motion planning for multiple car-like robots using probabilistic roadmaps. In Proceedings of ICRA'95, pages 1631-1636, Nagoya, Japan.

Tanner, H. G. and Kumar, A. (2005). Towards decentralization of multi-robot navigation functions. In Proceedings of ICRA'05, pages 4132-4137, Barcelona, Spain.

Thrun, S. (1998). Learning metric-topological maps for indoor mobile robot navigation. Artificial Intelligence, 99(1):21-71.

Ulrich, I. and Borenstein, J. (1998). VFH+: Reliable obstacle avoidance for fast mobile robots. In Proceedings of ICRA'98, pages 1572-1577, Leuven, Belgium.

Wilmarth, S. A., Amatoy, N. M., and Stiller, P. F. (1999). MAPRM: A probabilistic roadmap planner with sampling on the medial axis of the free space. In Proceedings of ICRA'99, pages 1024-1031, Detroit, MI, USA.

Wurm, K. M., Stachniss, C., and Burgard, W. (2008). Coordinated multi-robot exploration using a segmentation of the environment. In Proceedings of IROS'08, pages 1160-1165, Nice, France.

Zivkovic, Z., Bakker, B., and Kröse, B. (2006). Hierarchical map building and planning based on graph partitioning. In Proceedings of ICRA'06, pages 803-809, Orlando, FL, USA. 